\title{
Documentos
}

\section{CIRUGÍA BARIÁTRICA Y EMBARAZO}

\author{
Hugo Salinas $P^{1}{ }^{1}$, Benjamín Naranjo $D .^{1}$, Jorge Rojas $C{ }^{2}$, Beatriz Retamales M. ${ }^{\text {, Francisco }}$ \\ Vera $N^{b}$, Marco Sobrón B. ${ }^{b}$ \\ ${ }^{1}$ Departamento de Obstetricia y Ginecología, ${ }^{2}$ Departamento de Cirugía, Hospital Clínico de la Universidad de Chile.
}

anterna, bAlumnos, Escuela de Medicina, Universidad de Chile.

\section{RESUMEN}

El número de pacientes sometidos a cirugía bariátrica ha aumentado rápidamente en el mundo. Esta revisión pretende describir la literatura publicada relativa a las deficiencias nutricionales posteriores a la cirugía bariátrica y su importancia en el embarazo.

\section{PALABRAS CLAVES: Embarazo, cirugía bariátrica, obesidad mórbida}

\section{SUMMARY}

The number of patients with bariatric surgery has increased quickly on the world. This revision tries to describe the published literature relative to the nutritional deficiencies due to bariatric surgery and its importance in the pregnancy.

\section{KEY WORDS: Pregnancy, bariatric surgery, morbid obesity}

\section{INTRODUCCIÓN}

En Chile se han producido profundas modificaciones en el perfil epidemiológico de la población. En la última década la situación nutricional se ha modificado drásticamente, con un rápido aumento en la prevalencia de la obesidad en todos los grupos etáreos $(1,2)$. El cambio ha sido especialmente significativo en mujeres embarazadas; en sólo 15 años la prevalencia de obesidad se ha duplicado llegando cifras superiores al $30 \%(2,3)$.

En la literatura, la obesidad se ha asociado a un mayor riesgo de preeclampsia, diabetes gestacional, macrosomía fetal y cesárea (4-8), además de mayor ocurrencia de complicaciones durante el embarazo, parto y postparto (9-14).

A nivel mundial, ha ido en aumento el número de mujeres obesas en edad fértil en que se considera la cirugía bariátrica, apareciendo ciertas in- certidumbres relacionadas con el embarazo luego de este tipo de cirugías. En nuestro país no existe una estimación del número de pacientes que se realizan esta intervención quirúrgica. No obstante, hoy en día llama la atención la presencia de un número no despreciable de pacientes embarazadas que fueron sometidas a este tipo de cirugía en el policlínico de obstetricia de nuestro hospital, motivo por el cual, hemos resuelto efectuar esta revisión para estar al tanto de la evidencia referente a las deficiencias nutricionales posteriores a la cirugía bariátrica y su potencial impacto en el embarazo.

\section{INDICACIONES DE CIRUGÍA BARIÁTRICA}

El tratamiento de primera línea para la obesidad mórbida es un programa médico que integra cambios de conducta, actividades físicas y apoyo 
psicológico. Sin embargo, en muchos casos, el tratamiento médico falla en conseguir una pérdida de peso sostenida y el tratamiento quirúrgico puede ser indicado en algunos casos específicos (IMC $>40$ ó >35 con comorbilidad). En este sentido, se ha establecido que el único tratamiento realmente efectivo para la obesidad mórbida es la cirugía bariátrica (15). En la Tabla I se enumeran las indicaciones de esta cirugía.

\section{TIPOS DE CIRUGÍA BARIÁTRICA}

Existen varias alternativas para la intervención quirúrgica, dentro de ellas se describen principalmente tres categorías de cirugía que conllevan una pérdida de peso importante (Tabla II). La primera categoría incluye procedimientos que son restrictivos propiamente tal. Estos procedimientos inducen pérdida de peso al reducir la capacidad gástrica, limitando drásticamente la ingesta de alimentos. La segunda categoría incluye procedimientos mixtos restrictivo-malabsortivos que además de reducir la capacidad gástrica, alteran la continuidad gástrica normal e interrumpen la absorción de los nutrientes y alimentos ingeridos. La tercera categoría incluye procedimientos exclusivamente malabsortivos; estos raramente son realizados, debido al alto riesgo de serias complicaciones en el tiempo (16). Cada categoría y procedimiento tiene sus propios resultados a largo plazo y su potencial impacto en la nutrición y el embarazo. Todas estas alternativas pueden resultar en deficiencias de hierro, vitamina B12, ácido fólico y calcio.

La cirugía de malabsorción restringe tanto la ingesta de alimento como la cantidad de calorías y

\section{Tabla I}

\section{INDICACIONES DE CIRUGÍA BARIÁTRICA (15)}

- IMC >40 o >35 con comorbilidades*

- Obesidad mórbida presente por más de 5 años

- Paciente sin historia de alcoholismo y otras drogodependencias o enfermedades psiquiátricas graves

- Pacientes entre 18 y 60 años

- Existencia de una adecuada comprensión de las alteraciones producidas por la intervención y una buena posibilidad de adhesión a la estrategia propuesta

\footnotetext{
* Diabetes Mellitus tipo 2, hipertensión arterial, arterosclerosis, dislipidemia, accidente cerebrovascular, patología biliar litiásica, osteoartritis, apnea del sueño, hipoventilación alveolar, incontinencia urinaria, reflujo gastroesofágico, infertilidad, algunos cánceres (colorrectal, riñón, mama, útero), etc.
}

Tabla II

PRINCIPALES TÉCNICAS QUIRÚRGICAS PARA EL TRATAMIENTO DE LA OBESIDAD MÓRBIDA

\begin{tabular}{ll}
\hline Técnicas & Cirugía \\
\hline 1. Restrictivas: & \\
& - gastroplastía vertical en banda \\
& - gastroplastía vertical anillada \\
& - banda gástrica ajustable \\
& - gastroplastía vertical reforzada \\
& - gastroplastía \\
& - gastroplastía vertical sin anillo
\end{tabular}

2. Malabsortivas:

- by-pass yeyunoileal

- switch duodenal

3. Mixtas:

- by-pass gástrico en $Y$ de Roux
- derivación biliopancreática (Sco-
pinaro, Marceau, Larrad)

nutrientes que el cuerpo absorbe. En este procedimiento, la pared gástrica es engrapada en el fondo, se secciona y se anastomosa un asa de yeyuno de aproximadamente $150 \mathrm{~cm}$ en $Y$ de Roux al saco gástrico remanente cerca de la unión gastroesofágica. Así, los contendidos gástricos drenan directamente en el yeyuno distal, saltando el estómago distal, duodeno y yeyuno proximal. Esta cirugía se ha asociado a déficit de absorción de hierro, vitamina B12 y deficiencias de vitaminas liposolubles, así como también, a anormalidades electrolíticas (17).

Las cirugías restrictivas incluyen la gastroplastía a banda vertical y gastroplastía a banda ajustable. En la gastroplastía a banda vertical, la conservación de la continuidad gastroduodenal limita la deficiencia de micronutrientes. El bandeo gástrico ajustable produce saciedad precoz y disminuye el consumo de alimentos. Este procedimiento se realiza con mayor frecuencia por abordaje laparoscópico. Ajustes subsecuentes de la banda gástrica alteran el tamaño luminal y la cantidad de alimento consumido (18).

Cuando se consideran los beneficios del bypass gástrico en el embarazo, la mayoría de los reportes han sido alentadores. La información disponible indica que estas mujeres tendrían menor riesgo de diabetes gestacional, síndrome hipertensivo del embarazo, macrosomía y de parto cesárea, al compararlas con las obesas que no se someten a esta cirugía $(18,19)$.

Es fundamental para el obstetra comprender las diferencias de cada procedimiento de manera 
de poder ofrecer los mejores cuidados y evitar las potenciales complicaciones. Dentro de los procedimientos quirúrgicos realizables, tres son los más comúnmente realizados y se presentan en la Figura 1.

\section{DEFICIENCIAS NUTRICIONALES PRODUCIDAS POR LA CIRUGÍA BARIÁTRICA}

Muchos pacientes obesos ya presentan deficiencias nutricionales clínicas o subclínicas antes de la cirugía, ya sean de vitamina $D$, folatos y vitamina B12. Por esta razón, deberían ser evaluados los niveles séricos de vitaminas y minerales antes de la cirugía, para evitar o minimizar las complicaciones nutricionales en el período postquirúrgico.

Conceptualmente, las deficiencias nutricionales específicas son menos frecuentes en los procedimientos restrictivos que en los malabsortivos $(20,21)$. No obstante, en los pacientes sometidos a cirugías restrictivas se observan frecuentemente la conducta de menor ingesta, la intolerancia a la comida y restricción de alimentos $(22,23)$. Indudablemente, se hacen necesarios seguimientos a largo plazo para prevenir o detectar deficiencias nutricionales en esos pacientes.

Los procedimientos malabsortivos son los que más se relacionan con desórdenes nutricionales. Generalmente, los procedimientos más agresivos conducen a una mayor incidencia de deficiencias nutricionales $(24,25)$.
Los principales nutrientes afectados por la cirugía son las proteínas, vitamina B12 y folatos, hierro y calcio. Por este motivo, debería incorporarse una exploración cuidadosa y regular de estos nutrientes una vez que sus deficiencias limiten la calidad de vida.

\section{Déficit calórico-proteico}

El objetivo de la cirugía bariátrica es la restricción de la disponibilidad de energía exógena. Déficit calóricos suceden como consecuencia de una baja ingesta de alimento, intolerancia por algunos alimentos (principalmente de carne y lácteos) o malabsorción de nutrientes (principalmente lípidos y proteínas). La razón entre pérdida de masa grasa y libre de grasa es alrededor de 4:1 en las técnicas restrictivas $(20,26,27)$.

En algunos estudios la derivación biliopancreática aparece como causante de severas deficiencias calórico-proteicas $(28,29)$, mientras que en otros, se describen bajas incidencias $(30,31)$.

\section{Hierro}

Su déficit es común luego de una cirugía de by-pass gástrico y se relaciona parcialmente con una menor ingesta de adecuadas cantidades de carne y otros alimentos ricos en este oligoelemento. Además, los cambios anatómicos producidos por la cirugía previenen que los alimentos que lo contienen se expongan al ambiente ácido del

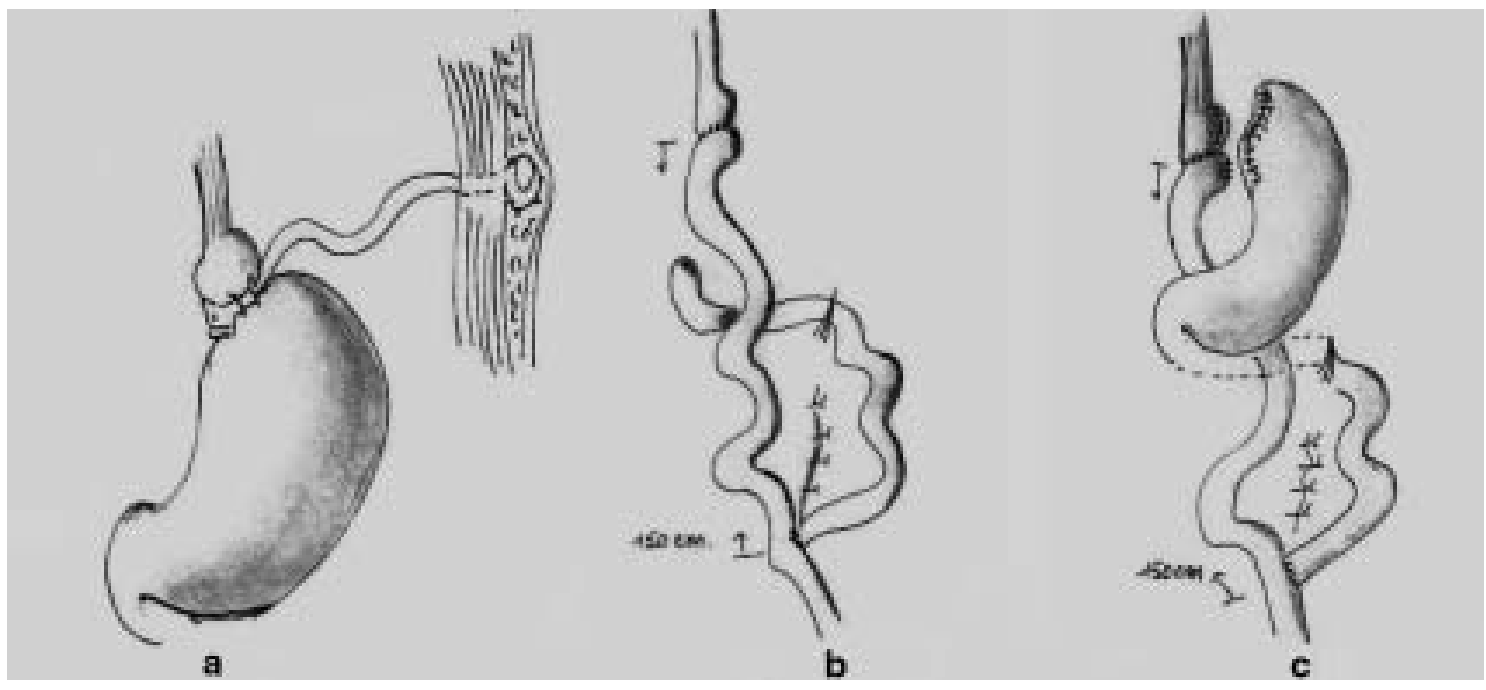

Figura 1. Procedimientos quirúrgicos de cirugía bariátrica más frecuentemente realizados en el Hospital Clínico de la Universidad de Chile. a) Banda gástrica ajustable, b) By-pass gástrico en $Y$ de Roux con sección gástrica, c) By-pass gástrico en $Y$ de Roux sin sección gástrica. (Dibujos del Dr. Jorge Rojas C.). 
estómago, requerido para su liberación desde la fuente proteica. A su vez, la acidez gástrica es esencial para la reducción de su forma férrica a ferrosa, que es necesaria para su absorción. Aunque al saltar el paso por el duodeno y el yeyuno proximal, se excluye el sitio primario para su absorción, algo de este se absorberá en el yeyuno distal. Se recomienda, luego de un by-pass gástrico que se adicione 40 a $65 \mathrm{mg}$ de sal ferrosa como suplemento diario. Sin embargo, individuos con una deficiencia preexistente o aquellos que sufrieron pérdidas sanguíneas, pueden requerir dosis más altas para mantener niveles adecuados (32). Mujeres sometidas a procedimientos puramente restrictivos, en los que la continuidad del tubo digestivo no se interrumpe, generalmente no sufren deficiencias de hierro ni se requiere suplementación de rutina (33).

\section{Vitamina B12}

La deficiencia de cobalamina, aunque menos común que la de hierro, ocurre en forma importante luego de un by-pass gástrico a no ser que se administren suplementos. El flujo digestivo normal permite que los alimentos sean degradados por el ácido gástrico para liberar vitamina B12 desde su fuente alimenticia. Entonces, la vitamina B12 no ligada se ensambla a ligandos $R$, que son glicoproteínas que se encuentran en las secreciones digestivas. Estos son degradados normalmente en el duodeno por proteasas pancreáticas, que permiten que la vitamina B12 liberada se combine con factor intrínseco (FI).

El FI es producido por las células parietales situadas en el fondo del estómago. Este complejo Fl/B12 está en definitiva listo para ser absorbido en el íleon distal. Sin embargo, luego de una cirugía de by-pass gástrico, la ausencia de un ambiente ácido no sólo evita la liberación de vitamina B12 de los alimentos, sino que también, inhibe la unión y subsecuente liberación entre cobalamina y ligandos $R$. Una reducción en la disponibilidad de $\mathrm{FI}$ combinada con la una menor presencia de la vitamina B12 no ligada previene la formación del complejo Fl/B12, dando como resultado la malaabsorción y la subsecuente deficiencia de cobalamina (32). Las mujeres sometidas a esta cirugía que presentan deficiencia de vitamina B12 a menudo presentan además niveles séricos elevados de homocisteína. La necesidad vital de niveles adecuados de vitamina B12 en la degradación de la homocisteína y la relación entre hiperhomocistinemia y anormalidades cardiovasculares y neurológicas refuerza la necesidad de suplementación a largo plazo luego de una cirugía bariátrica $(34,35)$. Dosis diarias de 350 ug de vitamina B12 en la forma cristalina son generalmente suficientes para mantener niveles séricos adecuados de cobalamina que normalizarán la homocisteína sérica. En los casos en que la suplementación oral no es adecuada, la terapia parenteral mensual puede ser la alternativa $(32,35)$.

\section{Folatos}

Los folatos (ácido folico, vitamina B9, folacina, y ácido tetrahidrofólico) son esenciales para la producción de hemoglobina y la regulación del desarrollo neuronal en el embrión y el feto en crecimiento $(36,37)$. Después de la cirugía de bypass gástrico, los alimentos ricos en ácido fólico saltan el duodeno, que es el sitio primario para su absorción. Sin embargo, ocurren ciertas adaptaciones del organismo que permiten que pequeñas cantidades de ácido fólico sean absorbidas del intestino delgado inferior (16). La suplementación prenatal con vitaminas que contienen $1 \mathrm{mg}$ de ácido fólico antes y durante del embarazo es generalmente suficiente para mantener los niveles adecuados y reducir el riesgo para defectos del tubo neural en el feto en desarrollo (32). La deficiencia crónica de folato o de vitamina B12 puede conducir a anemia megaloblástica. Dado que estas vitaminas son necesarias para la maduración de los glóbulos rojos, una deficiencia de cualquiera de las dos puede dar lugar a la liberación de glóbulos rojos inmaduros (o reticulocitos) al flujo sanguíneo circulante. Generalmente, con la suplementación de vitamina $B$ se observa una mejoría rápida. Cuando la anemia persiste, la presencia de esta condición debe ser considerada después de cirugía de by-pass gástrico (37).

\section{Calcio y vitamina D}

La deficiencia de calcio producida por una cirugía de by-pass gástrico es frecuente y puede resultar de una ingesta inadecuada o de malabsorción. Normalmente, el calcio junto con la vitamina D como mediador, se absorbe en el duodeno. Luego de un by-pass gástrico, se evita este sitio de absorción y los pacientes con esta cirugía, absorben el calcio a través de difusión pasiva a lo largo del remanente de intestino delgado, mecanismo que es dependiente del aumento de ingesta de alimentos (32). Debido a las propiedades del metabolismo óseo, la medición del calcio sérico puede no 
revelar exactamente el déficit de estos nutrientes. Las fracturas óseas pueden ser la primera señal de deficiencia de calcio. Hay que tener cuidado de prescribir el calcio en la forma de citrato, pues no requiere un ambiente ácido para ser degradado y ser absorbido. El suplemento diario con 1200 a $1500 \mathrm{mg}$ de citrato de calcio con vitamina $D$ se recomienda para que los pacientes operados prevengan la deficiencia (16). La Tabla III $(16,32)$ resume los suplementos recomendados de vitamina y minerales para los pacientes portadores de by-pass gástrico.

\section{Tiamina}

Una de las principales funciones de la tiamina es su participación en el metabolismo de los carbohidratos. Su absorción ocurre en todo el duodeno, principalmente en el entorno ácido del duodeno proximal. La deficiencia ocurre por la combinación de una reducción de la producción de ácido por el estómago, restricción de ingesta dietaria y episodios frecuentes de vómitos $(38,39,40)$. Altos ingresos de carbohidratos podrían precipitar síntomas clínicos en pacientes con depósitos bajos de tiamina $(41,42)$.

Conforme a que la ingesta de productos multivitamínicos puede prevenir deficiencias de tiamina en la mayoría de los casos, la administración de 50-100 mg de tiamina debiera corregir la deficiencia en los casos en que se sospecha. En pacientes con hiperemesis, la administración de tiamina 6 semanas post cirugía debería prevenir la deficiencia $(41,42)$.

\section{Otras deficiencias de vitaminas liposolubes y minerales}

Se han descrito otras deficiencias de vitaminas liposolubes y minerales (vitamina $\mathrm{E}, \mathrm{A}, \mathrm{K}$ y Zinc) (43-50), especialmente posterior a una derivación biliopancréatica. Basado en sus resultados, se recomienda un control sérico de vitaminas liposolubles antes y de manera regular post derivación biliopancreática.

\section{CONCLUSIÓN}

Las complicaciones nutricionales son comunes después de una cirugía bariátrica. Dependiendo del tipo de cirugía se producen predominantemente uno $y / u$ otro tipo de deficiencia. Durante el embarazo y como consecuencia de las deficiencias nutricionales, la madre y el feto en desarrollo
Tabla III

\section{RESUMEN DE RECOMENDACIONES DE SUPLEMENTO NUTRICIONAL}

\begin{tabular}{ll}
\hline Suplemento & Dosis \\
Ácido fólico & $1 \mathrm{mg}$ (en vitaminas prenatales) \\
Vitamina B12 & $350-500 \mathrm{ug} / \mathrm{día}$ (forma cristalina) \\
Calcio & $1200-1500 \mathrm{mg}$ (como citrato) \\
Sulfato o glu- & $325-650 \mathrm{mg} /$ día (40-65 mg) \\
conato ferroso & \\
Vitamina D & 50,000 unidades/día \\
Proteínas & $40-100 \mathrm{mg} /$ día
\end{tabular}

pueden presentar serias complicaciones a corto y largo plazo. El seguimiento postoperatorio habitual con suplemento de vitaminas y minerales previo a la ocurrencia de un embarazo sería lo ideal. No obstante, dado que algunas embarazadas se pierden del seguimiento, durante el primer control prenatal, es responsabilidad del obstetra prestar atención y realizar la prevención y tratamiento de las deficiencias nutricionales, además de educar a las pacientes sobre los riesgos potenciales que pueden ocurrir en el embarazo.

Luego de una cirugía bariátrica, se recomienda que todas las mujeres embarazadas se suplementen con vitaminas y minerales prenatales en forma diaria considerando los factores individuales de cada una de ellas. Según la evidencia disponible en la literatura, un control y manejo adecuado de las gestantes que fueron sometidas a cirugía bariátrica, parece ser el factor fundamental para lograr los mejores resultados obstétricos.

\section{BIBLIOGRAFÍA}

1. Albala C, Vio F, Kain J, Uauy R. Nutrition transition in Chile: determinants and consequences. Public Health Nutr 2002;5:123-8.

2. Uauy R, Atalah E, Kain J. The nutritional transition: new nutritional influences on child growth. In: Nutrition and growth. Martorell R, Haschke F. (eds). Williams \& Wilkins, Philadelphia, USA, 200;305-28.

3. Mardones F. Evolución de la antropometría materna y del peso de nacimiento en Chile, 1987-2000. Rev Chil Nutr 2003;30:122-31.

4. Weiss JL, Malone FD, Emig D, Ball RH, Nyberg DA, Comstock $\mathrm{CH}$, et al. Obesity, obstetric complications and cesarean delivery rate-a population-based screening study. FASTER Research Consortium. Am J Obstet Gynecol 2004;190:1091-7.

5. Baeten JM, Bukusi EA, Lambe M. Pregnancy complications and outcomes among overweight and 
obese nulliparous women. Am J Public Health 2001;91:436-40.

6. Young TK, Woodmansee B. Factors that associated with cesarean delivery in a large private practice: the importance of prepregnancy body mass index and weight gain. Am J Obstet Gynecol 2002;187:312-8.

7. Sebire NJ, Jolly M, Harris JP, Wadsworth J, Joffe M, Beard RW, et al. Maternal obesity and pregnancy outcome: a study of 287.213 pregnancies in London. In J Obes Relat Metab Disord 2001;25:1175-82.

8. Cedergren MI. Maternal morbid obesity and the risk of adverse pregnancy outcome. Obstet Gynecol 2004;103:219-24.

9. Brockelsby J, Dresner M. Obesity and pregnancy. Curr Anaest Crit Care 2006. In Press. DOI:10.1016/ j.cacc.2006.02.010.

10. Perlow JH, Morgan MA. Massive maternal obesity and perioperative cesarean morbidity. Am J Obstet Gynecol 1994;170:560-5.

11. Myles TD, Gooch J, Santolaya J. Obesity as an independent risk factor for infectious morbidity in patients who undergo cesarean delivery. Obstet Gynecol 2002;100:959-64.

12. Kabiru W, Raynor BD. Obstetric outcomes associated with increase in BMl category during pregnancy. Am J Obstet Gynecol 2004;191:928-32.

13. Robinson HE, O'Connell CM, Joseph KS, McLeod NL. Maternal outcomes in pregnancies complicated by obesity. Obstet Gynecol 2005;106(6):1357-64.

14. Robinovitch J, Rubio E, Sáez J, Ramírez M. Influencia del peso corporal en el embarazo y resultado perinatal. Rev Chil Obstet Ginecol 1995;60:151-67.

15. NIH. Consensus development conference panel. Gastrointestinal surgery for sever obesity. Ann Intern Med 1991;115:956-61.

16. Elliot K. Nutritional considerations alter bariatric surgery. Crit Care Nurs Q 2003;26:133-8.

17. Gurewithsch ED, Smith-Levitin M, Mack J. Pregnancy following gastric bypass surgery for morbid obesity. Obstet Gynecol 1996;88:658-61.

18. Martin LF, Finigan KM, Nolan TE. Pregnancy after adjustable gastric banding. Obstet Gynecol 2000;95:927-30.

19. Richards DS, Miller DK, Goodman GN. Pregnancy after gastric bypass for morbid obesity. J Reprod Med 1987;32:172-6.

20. Giusti V, Suter M, Heraief E, et al. Effects of laparoscopic gastric banding on body composition, metabolic profile and nutritional status of obese women: 12-months follow-up. Obes Surg 2004; 14:239-345.

21. Chapman AE, Kiroff G, Game P, et al. Laparoscopic adjustable gastric banding in the treatment of obesity: a systematic literature review. Surgery 2004;135(3):326-51.

22. Shai I, Henkin Y, Weitzman S, Levi I. Long-term dietary changes after vertical banded gastroplasty: is the trade-off favorable? Obes Surg 2002;12:805-11.
23. Kriwanek S, Blauensteiner W, Lebisch E, et al. Dietary changes after vertical banded gastroplasty. Obes Surg 2000;10:37-40.

24. Brolin RE, Leung M. Survey of vitamin and mineral supplementation after gastric bypass and biliopancreatic diversion for morbid obesity. Obes Surg 1999;9:150-4.

25. Marcason W. What are the dietary guidelines following bariatric surgery? J Am Diet Assoc 2004;104: 487-8.

26. Strauss BJ, Marks SJ, Growcott JP, Stroud DB, Lo CS, Dixon JB, et al. Body composition changes following laparoscopic gastric banding for morbid obesity. Acta Diabetol 2003;40 (Suppl 1):S266S269.

27. Moize V, Geliebter A, Gluck ME, et al. Obese patients have inadequate protein intake related to protein intolerance up to 1 year following Roux-en-Y gastric bypass. Obes Surg 2003;13:23-8.

28. Sugerman HJ. Bariatric surgery for severe obesity. $J$ Assoc Acad Minor Phys 2001;12:129-36.

29. Benedetti G, Mingrone G, Marcoccia S, et al. Body composition and energy expenditure after weight loss following bariatric surgery. J Am Coll Nutr 2000; 19:270-4.

30. Totte E, Hendrickx L, van Hee R. Biliopancreatic diversion for treatment of morbid obesity: experience in 180 consecutive cases. Obes Surg 1999;9:161-5.

31. Scopinaro N, Adami GF, Marinari GM, et al. Biliopancreatic diversion. World J Surg 1998;22:936-46.

32. Kushner R. Managing the obese patient after bariatric surgery: a case report of severe malnutrition and review of the literature. JPEN J Parenter Enteral Nutri 2000;24:126-32.

33. Dixon JB, Dixon ME, Obrien PE. Pregnancy after lapband surgery: management of the band to achieve healthy weight outcomes. Obes Surg 200;11:5965.

34. Dixon JB, Dixon ME, Obrien PE. Pregnancy after lapband surgery: management of the band to achieve healthy weight outcomes. Obes Surg 2001;11:5965.

35. Sumner AE, Chin MM, Abrahm JL, et al. Elevated methylmalonic acid and total homocysteine levels show high prevalence of vitamin B12 deficiency after gastric surgery. Ann Intern Med 1996;124:469-76.

36. Picciano MF. Pregnancy and lactation: physiological adjustments, nutritional requirements and the role of dietary supplements. J Nutr 2003; 133(6):1997S2002S.

37. Chandra J, Jain V, Narayan S, et al. Folate and cobalamin deficiency in megaloblastic anemia in children. Indian Pediatr 2002;39:453-57.

38. Loh Y, Watson WD, Verma A, et al. Acute Wernicke's encephalopathy following bariatric surgery: clinical course and MRI correlation. Obes Surg 2004;14: 129-32.

39. Coskun H, Bozbora A, Ogunc G, Peker Y. Adjustable 
gastric banding in a multicenter study in Turkey. Obes Surg 2003;13:294-6.

40. Houdent C, Verger N, Courtois H, et al. Wernicke's encephalopathy after vertical banded gastroplasty for morbid obesity. Rev Med Interne 2003;24:476-7.

41. Sola E, Morillas C, Garzon S, et al. Rapid onset of Wernicke's encephalopathy following gastric restrictive surgery. Obes Surg 2003;13:661-2.

42. Chaves LC, Faintuch J, Kahwage S, Alencar Fde A. A cluster of polyneuropathy and Wernicke-Korsakoff syndrome in a bariatric unit. Obes Surg 2002;12: 328-34.

43. Scopinaro N, Marinari GM, Pretolesi F, et al. Energy and nitrogen absorptionafter biliopancreatic diversion. Obes Surg 2000;10:436-41.

44. Trostler N, Mann A, Zilberbush N, et al. Nutrient intake following vertical banded gastroplasty or gastric bypass. Obes Surg 1995;5:403-10.

45. Vazquez C, Morejon E, Munoz C, et al. Nutritional effect of bariatric surgery with Scopinaro operation.
Analysis of 40 cases. Nutr Hosp 2003;18:189-93.

46. Kisakol G, Guney E, Bayraktar F, et al. Effect of surgical weight loss on free radical and antioxidant balance: a preliminary report. Obes Surg 2002;12: 795-801.

47. Spits Y, De Laey JJ, Leroy BP. Rapid recovery of night blindness due to obesity surgery after vitamin A repletion therapy. Br J Ophthalmol 2004;88:583-5.

48. Hatizifotis M, Dolan K, Newbury L, Fielding G. Symptomatic vitamin A deficiency following biliopancreatic diversion. Obes Surg 2003;13:655-7.

49. Quaranta L, Nascimbeni G, Semeraro F, Quaranta CA. Severe corneoconjunctival xerosis after biliopancreatic bypass for obesity (Scopinaro's operation). Am J Ophthalmol 1994;118:817-8.

50. Huerta S, Rogers LM, Li Z, et al. Vitamin A deficiency in a newborn resulting from maternal hypovitaminosis $\mathrm{A}$ after biliopancreatic diversion for the treatment of morbid obesity. Am J Clin Nutr 2002;76: 426-9. 